\title{
Prédestination ou prédétermination dans l'islam ?
}

De Friedrich Ulrich à Max Weber

\section{Youcef Djedi}

\section{(2) OpenEdition}

\section{Journals}

Édition électronique

URL : http://journals.openedition.org/ress/1065

DOI : $10.4000 /$ ress. 1065

ISSN : 1663-4446

Éditeur

Librairie Droz

\section{Édition imprimée}

Date de publication : 15 décembre 2011

Pagination : 259-274

ISBN : 978-2-600-01575-2

ISSN : 0048-8046

\section{Référence électronique}

Youcef Djedi, « Prédestination ou prédétermination dans l'islam ? », Revue européenne des sciences sociales [En ligne], 49-2 | 2011, mis en ligne le 01 janvier 2015, consulté le 19 avril 2019. URL : http:// journals.openedition.org/ress/1065; DOI : 10.4000/ress.1065 


\title{
PRÉDESTINATION OU PRÉDÉTERMINATION DANS L'ISLAM?
}

\author{
DE FRIEDRICH ULRICH À MAX WEBER
}

YOUCEF DJEDI

Université de Versailles

youcef.djedi@uvsq.fr

\begin{abstract}
Résumé. Dans ses quelques traitements de la doctrine islamique de la prédestination, Max Weber se réfère à la thèse du pasteur luthérien Friedrich Ulrich sur la prédestination dans l'islam et le christianisme. Or l'un des moments forts de cette thèse consiste précisément à distinguer entre «prédestination» proprement dite et «prédétermination », cette dernière caractérisant alors davantage la conception islamique, tenue pour plus faiblement sotériologique. Consolidée par la caution wébérienne, pour ainsi parler, cette idée réapparaît de temps en temps, sans examen, pour réitérer les mêmes arguments hâtifs. Pourtant, des travaux à l'époque même de Weber, et qui plus est étaient connus de Friedrich Ulrich, permettent de voir à quel point l'activité missionnaire de celui-ci avait guidé sa recherche.
\end{abstract}

Mots-clés: ascétisme, double décret, islam, prédestination, prédétermination, sotériologie

\begin{abstract}
When dealing with the Islamic predestination, Max Weber refers several times to the dissertation of the Lutheran minister Friedrich Ulrich about the Islamic and Christian predestination dogma. Yet this research went to the distinction between true "predestination" and "predetermination", the latter more appropriated to the Islamic conception, since it is allegedly less concerned with salvation. Because of the authority of Max Weber, this idea is still sometimes reiterated with the same superficial arguments. Yet, there were researches even at the time of Weber (and which were well known by Friedrich Ulrich), which could show by contrast how important was the part of the Evangelic missionary activity of the latter in the conception of his dissertation.
\end{abstract}

Keywords : asceticism, double decree, Islam, predestination, predetermination, salvation 
Dans son étude sur L’Éthique protestante et l'esprit du capitalisme, Max Weber assigne à la doctrine de la prédestination un rôle des plus significatifs dans la formation de cette «conduite de vie» si spécifique au protestantisme ascétique. En résumé, les calvinistes adeptes du decretum horribile n’inclinaient pas seulement à la certitude d’être les «élus », mais se faisaient aussi de la persévérance dans leur état de sainteté un souci quotidien. En insistant sur l'inséparabilité de la foi et des œuvres, le calvinisme avait pavé la voie vers la confirmation de l'état de grâce à l'intérieur d'un métier.

Bien sûr, comme l'indiquera encore Weber lui-même ailleurs, cette doctrine, plus ancienne que le protestantisme, n'est pas même propre au christianisme:

Dans l'islamisme, les fidèles les plus passionnés d'Allâh lui ont attribué cette toute-puissance absolue; de même les virtuoses chrétiens l'ont attribuée au deus absconditus. Un décret de Dieu, décret souverain, libre, absolument insondable et, conséquence logique de son omniscience, établi depuis toujours, a décidé de la destinée des hommes sur terre et après leur mort. La détermination de la destinée terrestre ainsi que la prédestination dans l'autre monde sont fixées de toute éternité. Les animaux pourraient déplorer tout autant de ne pas avoir été créés hommes que les damnés de voir leur réprobation fixée par la prédestination. (Weber M., 200I, I, 22 / 2, p. 260).

Et comme il le montre lui-même, la «conduite de vie» ascétique, et ses retombées sur l'essor du capitalisme moderne, n'étaient pas exclusives à cette croyance en la double prédestination: l'anabaptisme (et ses diverses ramifications) a été porteur d'un ascétisme aussi remarquable - sinon plus - que celui du calvinisme, alors qu'il refusait le plus souvent toute référence à la prédestination. Mais, du point de vue de l'«histoire culturelle universelle», le plus important à retenir concernant la doctrine calviniste du double décret, c'est surtout que, pour Max Weber, le rôle joué par celle-ci dans la connexion entre biens «intramondains » et bénéfices sotériologiques ne s'était trouvé nulle part ailleurs.

Cette considération disqualifie l'islam, qui avait développé, lui aussi, une croyance en la prédestination. Max Weber connaissait l'existence de ce dogme dans l'islam, mais force est de reconnaître que certaines de ses affirmations à ce propos sont sujettes à caution. Certes, on ne contestera guère son insistance sur la réalité du fatalisme dans lequel baignaient les masses musulmanes à son 
époque. Mais cela était-il vraiment dû à la doctrine de la prédestination? N’y avait-il pas plutôt, comme l'avait souligné son ami et collègue, Carl Heinrich Becker, une relation directe entre le déclassement social et économique des musulmans et leur conception du monde devenue fataliste?

Il est vrai, comme l'explique Weber lui-même, que, lorsque la doctrine de la prédestination se vide de son acception de double décret proprement dit, elle peut se transformer en fatalisme. Mais cela supposerait alors aussi l'absence de tout «providentialisme » et de toute idée de vie future, ce qui est loin d'être le cas en islam. En fait, selon Weber, le fatalisme musulman découlerait plutôt du fait même que la prédestination islamique soit en vérité un prédéterminisme. Notre sociologue suit ici, comme il l'indique lui-même, les conclusions auxquelles aboutit le pasteur Friedrich Ulrich, dans sa thèse de théologie sur La prédestination dans l'islam et le christianisme (Ulrich F., I9I2).

\section{FRIEDRICH ULRICH ET SA THĖSE SUR LA PRÉDESTINATION DANS L'ISLAM ET LE CHRISTIANISME}

En tant que recherche théologique portant sur un point de doctrine islamique, la thèse de Friedrich Ulrich est de peu d'originalité et ne contraste pas vraiment avec les écrits du genre à l'époque. Mais cela ne l'a pas empêché de se frayer parfois une place dans des contextes wébériens sur l'islam - ou même pas nommément wébériens, ni non plus en rapport direct avec la prédestination. Pourtant, conçue en une période d’affrontement plus religieux que politique, elle montre en plusieurs endroits les présupposés « missionnaires » qui l'avaient alimentée. D’autant plus, du reste, qu'en la confrontant à des travaux de la même époque sur le même sujet, on voit clairement à quel point son auteur - et Max Weber à sa suite - s'était fourvoyé en présentant la prédestination islamique comme une simple prédétermination.

Sur Friedrich Ulrich, nous ne savons rien d'autre que ce que contient le bref Lebenslauf, inséré à la fin de sa thèse, selon l'usage allemand. Suivant ce curriculum vitae, cet auteur était pasteur à Sarrebruck. Mais la future capitale de la Sarre n’ayant pas d'université, cela explique peut-être pourquoi notre homme avait 
préparé sa thèse à l'université de Heidelberg, bien qu'à l'époque Strasbourg, devanture des études orientales de l'Allemagne wilhelmienne, fût de prime abord plus près de Sarrebruck. Mais la ville de Max Weber, qui abritait depuis les premières décennies du seizième siècle une chaire d’arabe, était connue, elle aussi, et depuis très longtemps, pour ses activités dans la recherche sur l'islam.

Le même curriculum vitae nous indique également que l'activité missionnaire du pasteur Ulrich était concentrée, avant 1912, au Moyen-Orient, notamment au Liban. On est donc en droit de supposer que le sujet de sa recherche avait un rapport manifeste avec son activité de missionnaire en terre d'islam. Pourtant, son intérêt pour l'islam ne semble pas avoir suscité d’autres écrits que cette thèse. En effet, sauf à avoir publié dans des revues de missions, par exemple, on ne lui connaît pas d'autres écrits ayant traité de cette religion. Pour le reste, Ulrich ne semble avoir signé, avant I9ı2, qu’un petit pamphlet sur le néo-protestantisme (Ulrich F., I9Io), et le plus gros de ce qu'il a écrit par la suite reflète plutôt ses préoccupations paroissiales caritatives (Ulrich F, 1925; 1929; 1932) et théologiques protestantes (Ulrich F., 1934; 1935), sans plus aucun rapport avec l'islam.

Une présentation de Die Vorherbestimmungslehre im Islam und Christentum a déjà été faite par Wolfgang Schluchter dans son introduction de Max Webers Sicht Des Islams (Schluchter W., 1987, p. 36 et suiv.). Mais il nous a semblé opportun de donner ici au lecteur non germanophone un résumé de la conclusion dans laquelle Ulrich reprend les principaux moments de sa recherche.

Pour ce pasteur luthérien, l'islam ne connaît pas autre chose que la souveraineté et la toute-puissance d'Allâh, qui devient ainsi une sorte de despote, mais sans que cela n’engendre un sentiment de «vénération». La relation à Dieu prend la forme d'une relation de sujet à souverain et non pas d'enfant à père, alors que le christianisme n’a jamais conçu de façon aussi mécanique les rapports de l'homme avec Dieu, pas même dans ses formulations les plus poussées de l’absoluité divine, comme c'était le cas chez Calvin', par exemple.

I On se souviendra que c'était précisément là l'une des principales critiques faites au calvinisme par Leibniz... 
À vrai dire, ce qui fait défaut à l'islam, selon Friedrich Ulrich, c'est l'absence d'une personnalité médiatrice de Sauveur, car cette religion strictement monothéiste, n'oserait donner ce rôle à aucun mortel, pas même au Prophète. Or justement, cette déficience ne pouvait qu’accentuer la faiblesse déjà manifeste des rapports proprement éthiques entre Allâh et ses créatures. Et la conséquence d'une carence aussi importante réside aussi dans l'absence de l'idée de «personnalité », que le christianisme, au contraire, a su sauvegarder, parce que ses fondements sont dans la foi et l'amour, et non pas, comme chez les musulmans, dans la loi et l'obligation. Ulrich insiste aussi sur l'idée, selon laquelle l'islam ignore le péché proprement dit et ne connaît aucun traducianisme comme conséquence du péché originel ${ }^{2}$. D’où l’absence, d’après lui, d’un besoin spécifique de la grâce divine, dont le terminus technicus même serait inexistant en islam.

En revanche, il reconnaît que la religion musulmane laisse apparaître parfois une dissipation du déterminisme qui pèse sur les êtres et admet une place à la liberté et à la responsabilité humaines. Mais en cela l'islam n’a, d’après lui, aucun mérite, dès lors qu'on ne peut effacer de l'histoire religieuse et de l'âme humaine indéfiniment, ni complètement, des thèmes aussi profondément ancrés. L'évolution du calvinisme en est, s'il en faut, la preuve. Mais au fait - et c'est là le principal emprunt de Max Weber à cette thèse -,

peut-on seulement parler d'une doctrine islamique de la prédestination, entendue au sens que l'histoire dogmatique lui donne, c'est-à-dire dans une acception singulièrement sotériologique! [...] À bien voir donc, ce qui caractérise vraiment la doctrine islamique, c'est qu'elle renferme plutôt un prédéterminisme cosmologique [kosmologisch bestimmter Prädeterminismus], dans lequel prédomine, non pas [...] la conception d'une élection par la grâce [Gnadenwahl], mais une stricte dépendance vis-à-vis d'Allâh, dont la volonté absolue fixe la totalité du destin des individus. De prédestination il n'en est point, et c'est en vain que l'on chercherait dans le Coran l'immuable double décret sauvant les uns et réprouvant les autres. L'intérêt sotériologique - encore faudrait-il que l'islam eût d’abord

2 Doctrine chrétienne selon laquelle le péché et la mort se transmettent dans l'espèce adamique, depuis la faute du premier couple humain. D'où la nécessité d'une grâce qui sauve. Mais cette «chute» (lapsisme) avait-elle été prévue par la prédestination? Oui, disent les «supralapsistes», contrairement aux «infralapsistes». Dans l'islam, l'opinion générale est supralapsiste, bien que le Coran ne traite pas la faute d'Adam et d'Ève en termes de «chute» proprement dite. 
une doctrine du salut - s'efface, en conséquence, devant l'intérêt théologique et téléologique. D’où cette tendance fataliste de plus en plus prononcée dans la mentalité musulmane, tendance que le christianisme ne connaît évidemment pas. Bien au contraire. Même quand il en venait parfois à proclamer ouvertement le déterminisme, $[. .$.$] ce n'était jamais qu’en parallèle avec la liberté$ humaine. Et le calvinisme qui avait professé autrefois la sévère prédestination avait toujours montré en même temps une activité si puissante et une telle culture volontariste [Kultur des Willens] qu'on en avait rarement vu de semblables dans l'histoire. (Ulrich F, I9I2, p. I30-I31).

Pour Friedrich Ulrich, c'est ce hiatus infranchissable, rigoureusement maintenu entre Allâh et ses créatures, qui est à l'origine de l’aridité si symptomatique de la vie religieuse musulmane. De la même manière, le prédéterminisme et le fatalisme qui lui est corollaire ont, sans nul doute, leur part de responsabilité dans la torpeur intellectuelle dont sont frappés depuis longtemps les peuples musulmans.

Certes, conclut Ulrich, l'islam a pu obtenir de ses adeptes - et pour fataliste que soit sa doctrine de la prédestination - de grands sacrifices et une patience devant l'adversité aussi étonnante que remarquable. Mais de renouvellement de la vie morale et culturelle, point. Parce qu'il lui manque précisément l'esprit du christianisme, c'est-à-dire un contenu religieux éthique, alors que la doctrine de la prédestination ne s'est presque jamais maintenue dans le christianisme sans être, justement, constamment régulée par un pendant éthique.

\section{CONTEXTE ET SOURCES}

Sans doute Friedrich Ulrich n'avait-il nulle prétention de faire œuvre d'Islamwissenschaftler, à cette époque où les islamologues eux-mêmes, à l'instar des spécialistes des autres «sciences de la culture», débattaient encore du nom à donner à leur discipline (Djedi Y., 20I I). Ce qu'il avait entrepris, c'était en fait une «incursion», comme on en faisait souvent, pour montrer la supériorité «culturelle »-mais aussi sotériologique - du christianisme. L'un des exemples assez typiques à ce propos est probablement celui de Adolf von Harnack, dont la conception du christianisme, et de l'ascétisme monacal médiéval en particulier, avait beaucoup influencé les sociologues du protestantisme, Weber en tête. Dans son Lehrbuch der Dogmengeschichte (Harnack A. v., I964, II, p. 529-538), 
ce célèbre théologien protestant consacre à l'islam un chapitre édifiant, dont l'écho se fit entendre jusque dans les milieux islamologiques de l'époque (Hanisch L., 2000, p. I59), tant il lui permit de mieux mettre en évidence l'excellence religieuse et culturelle du christianisme.

En tout cas, sans vouloir revenir sur la part active et importante prise par les missions protestantes dans l'expansion coloniale en terre d'islam (Becker C.H., I967, II, p. 2 I I et suiv.; Miège J.-L., 1955, p. I53-I89; TibawiA., 1963, p. 137-182), on ne peut s'empêcher de voir précisément dans la thèse de Friedrich Ulrich un document bien caractéristique de cette période, où la «question de l'islam »- ou la «question orientale» (Hauri J., I88I, p. I) - était considérée aussi du point de vue missionnaire. C'est là un fait très justement souligné à l'époque par l'ami et collègue de Max Weber, Carl Heinrich Becker (Becker C. H., 1967, II, p. 2I I), qui était luimême très impliqué dans la question de l'expansionnisme européen. Quant à Weber, on sait qu'il était très attentif aux diverses activités missionnaires, jusqu'à la lointaine Chine (Weber M., 1988, I, I00 n. 3).

Pourtant, nous sommes peu de temps avant la Première Guerre mondiale, et l'Allemagne est le principal soutien de l'empire ottoman déclinant, dont elle réorganise l'armée. Le Kaiser ne tardera pas à se déclarer l'ami des Turcs et de l'islam, dont il tient la Porte pour l'unique représentant. C'était là aussi comme un écho à certains théoriciens de la Mitteleuropa, comme Ernst Jäckh, par exemple, aux côtés duquel Max Weber et Gustav von Schmoller, notamment, siègeront à la Commission pour la Mitteleuropa, fondée en 1916 par Friedrich Naumann. Jäckh et ses partisans mettaient en avant l'importance géostratégique européenne de l'empire ottoman et lui reconnaissaient, de ce fait, une place indiscutable dans cet ensemble qu'ils voyaient contrebalancer dans le proche avenir l'action des empires britannique et russe.

D’après la bibliographie citée dans sa thèse, Friedrich Ulrich ne devait pas être bon arabisant. Le plus gros de sa documentation est, en effet, dans les langues européennes. Pour le commentaire coranique, par exemple, il avait utilisé la version latine de Baydawi, éditée par Heinirich Leberecht Fleicher (Baydawi A., I846/I848). Or justement, Baydawi ne le cède en rien au reste des 
commentateurs du Coran, lorsqu'il s'agit de souligner l'importance du double décret de la prédestination. Il est vrai, en revanche, que le même double décret semble comme perdu parmi les traditions recueillies dans le Sahih de Buhari, que venait d'éditer, quelques années auparavant, Ludolf Krehl (Buhari M. i. I. I908), dont Ulrich avait utilisé aussi un article consacré précisément, tout comme celui de Bernhardt Spiess (Spiess B., I872), à la prédestination en islam (Krehl L., I873).

Cela peut paraître étonnant, mais à la décharge de notre pasteur, le thème de la prédestination, sous sa forme de double décret, était à cette époque encore très peu débattu par les ténors des études islamiques. Le grand Goldziher lui-même, dont Friedrich Ulrich invoque l’autorité, ne s'était jamais vraiment décidé sur ce point, jusqu’à la thèse du Néerlandais Abraham de Vlieger (Vlieger A. [de], 1902), publiée une décennie avant celle du pasteur de Sarrebruck, qui d’ailleurs l’avait utilisée. Goldziher en fit un compte rendu l'année même de sa parution (Goldziher I., 1902, p. 392-402) et la tint, trois années plus tard, dans une communication, destinée au «Congress of Arts and Science de Saint-Louis », sur «Les progrès de l'islamologie durant les trois dernières décennies », pour l'une des dates les plus marquantes de cette période (Goldziher I, 1905, p. 208).

Fort d'une documentation de première main, Abraham de Vlieger était tout simplement arrivé à l'opposé des conclusions de la thèse de Friedrich Ulrich. On peut donc dire que celui-ci s'était quelque peu hâté de se réjouir de sa communauté de vue avec Goldziher (Ulrich F., 1912, p.6I n. I). En tout cas, dire que le Coran n'enseigne pas la double prédestination, comme le prétend Ulrich, c'est, non seulement «faire violence à la simple littéralité » du livre saint de l'islam, comme le notait déjà depuis longtemps Bernhardt Spiess (Spiess B., I873, p. 4), mais c'est aboutir aussi à l'aberration, selon laquelle, «les plus célèbres théologiens musulmans n’ont rien compris au Coran. » (Vlieger A. [de], 1902, p. IO-II). On voit à quel point il est hasardeux de ne voir dans la doctrine islamique de la prédestination qu'un prédéterminisme mécanique confinant au fatalisme.

En fait, la thèse de Friedrich Ulrich s'inscrit dans la droite ligne de travaux comme ces deux thèses, largement utilisées dans la sienne, et qui sont aussi l'œuvre de pasteurs : Der Islam in seinem Einfluss auf das Leben seiner Bekenner [L’islam dans son impact sur la vie de ses fidèles] de Johannes Hauri (Hauri J., I88I), en poste 
à Davos en Suisse, et Der Einfluss des Islam auf das häusliche, sociale und politische Leben seiner Bekenner. Eine Culturgeschichtliche Studie [L'influence de l'islam sur la vie domestique, sociale et politique de ses fidèles. Une étude d'histoire culturelle] de Nathanael C. Pischon (Pischon C. N., I88I), en poste à la Preußische Gesellschaft d'Istanbul [Société prussienne d'Istanbul]. Les deux recherches avaient d'ailleurs été primées par la Haager Gesellschaft zur Vertheidigung des Christentums [Société de la Haye pour la défense du christianisme], qui avait lancé un concours, en ı878, autour de la double question de savoir quel rôle l'islam avait joué, et joue encore, dans la vie domestique, sociale et politique de ses fidèles, et, à partir de là, ce qu'il revient au christianisme de faire contre l'islam et les musulmans.

\section{LA RÉCEPTION DE LA THĖSE DE FRIEDRICH ULRICH PAR MAX WEBER ET SES LECTEURS}

Si la thèse du pasteur Friedrich Ulrich est aujourd'hui un tant soit peu connue, c'est très certainement grâce à Max Weber, qui y renvoie par deux fois dans l'édition posthume de L'Éthique protestante et l'esprit du capitalisme. Dans ces deux notes, insérées dans son analyse des conséquences volontaristes du double décret de la prédestination calviniste sur la conduite de vie puritaine, le sociologue explique a contrario les effets d’après lui fatalistes de la prédestination dans l'islam. Certes, comme il l'indique lui-même, le risque de fatalisme est inhérent à la nature même de la croyance dans la prédestination:

Melanchthon et Wesley en parlent, mais il est caractéristique que, dans les deux cas, on avait affaire à une combinaison avec une religiosité de la «foi» de caractère sentimental. Celle-ci ne connaissant pas l'idée rationnelle de la confirmation, cette conséquence, de fait, était dans la nature des choses. - [L'islam a connu ces conséquences fatalistes. Mais pour quelle raison? Parce que la prédestination islamique se rapportait aux destinées ici-bas et non au salut dans l'au-delà, et cela sur le mode du prédéterminisme et non de la prédestination; parce que, conséquemment, l'élément décisif au plan éthique, à savoir la «confirmation» en tant que prédestiné, ne jouait plus aucun rôle dans l'islam et (qu'à l'instar de la möra) il ne pouvait en découler qu'une intrépidité guerrière, mais pas de conséquences au plan d'une méthode de vie, lesquelles ne bénéficiaient pas de «récompense» religieuse. (Voir la thèse de théologie de F. Ulrich, Die Vorherbestimmungslehre im Islam und Christentum, Heidelberg, 1912.)] (Weber M., I988, I, p. 102 n. 2 ; id., 2003, p. I16 n. 38). 
Plus loin, Max Weber confirme derechef ce «prédéterminisme » qu'il attribue à l'islam, lorsqu'il passe en revue les différentes approches protestantes de la «conduite de vie » et leurs rapports à la prédestination. C'est naturellement le calvinisme qui lui semble, en la matière, le plus cohérent et psychologiquement le plus efficace. Mais concernant l'islam, Weber se suffit d'un renvoi en note:

Sur les effets tout différents de la doctrine de la prédestination (ou, plus exactement : de la prédétermination) islamique et ses fondements, voir la thèse de théologie de F. Ulrich, citée plus haut: Die Vorherbestimmungslehre im Islam und Christentum, Heidelber, 1912. (Weber M., I988, I, p. I28 n. I ; id., 2003, p. I5 I n. I8).

Et c'est à l'évidence à la thèse de Friedrich Ulrich que Weber pensait aussi en analysant, cette fois, dans Économie et société (Schluchter W., 1987, p. I I n. 84), la prédestination islamique. Pour lui, celle-ci, en effet,

ne connaissait pas le double décret: on n'osait pas attribuer à Allâh la prédestination à l'enfer, mais seulement le retrait de sa grâce et ainsi l'entrée dans l'inévitable errance [Irren]. Et, dans la mesure où, en vertu de son caractère de religion de guerriers, elle avait la coloration de la «moïra» hellénique, les éléments spécifiquement rationnels de la "gestion du monde" [Weltregiment], d'une part, et la détermination du destin religieux de l'individu dans l'au-delà, d'autre part, avaient été en même temps beaucoup plus faiblement développés. La conception prévalait que ce n'était pas le destin dans l'au-delà, qui était déterminé par la prédestination, mais celui extra-quotidien [ausseralltäglich] d'ici-bas. (On posait), par exemple, [...] la question de savoir si le combattant de la foi allait tomber ou pas dans le champ de bataille. Quant au destin futur de l'individu, il était suffisamment assuré par la simple foi en Allah et au Prophète et ne nécessitait, du moins d'après la conception la plus ancienne, aucune confirmation dans une direction de vie [Lebensführung] : un système rationnel de l'ascèse quotidienne était, dès le début, étranger à cette religion de guerriers. Ainsi, dans l'islam, la prédestination développait son pouvoir toujours de nouveau dans les batailles pour la foi [Glaubenskämpfen], comme encore dans celles du Mahdi, (mais) elle perdait, en revanche, avec chaque embourgeoisement de l'Islam, parce qu'elle ne fondait aucune méthodologie de vie intra-quotidienne [inneralltäglich], comme dans le puritanisme, où la prédestination concernait justement le destin dans l'au-delà et où la certitudo salutis dépendait donc de la confirmation intra-quotidienne de la vertu [inner alltäglichen Tugendbewährung]. C'est pourquoi, c'était seulement avec l'embourgeoisement de la religiosité de Calvin que sa signification, à l'égard des propres vues originelles de celui-ci, augmenta. (Weber M., 200I, I/22-2, p. 364-366). 
Il faut noter que la thèse de Friedrich Ulrich était manifestement passée inaperçue et que, jusqu'à plus ample informé, elle n'avait fait l'objet d'aucun compte rendu dans les revues de l'époque. Aucune mention n'en est faite dans la correspondance entre les grands islamologues contemporains de cette publication. On peut donc avancer, sans grand risque d'erreur, que Max Weber n’avait eu connaissance de cette thèse que parce qu'elle avait été soutenue à Heidelberg, à l'université même où il était professeur.

Ce qui est sûr toutefois, c'est que le destin de cette publication avait été assuré par la caution, pour ainsi dire, que lui avait apportée Max Weber en la citant expressément à deux reprises. Ce point est d'autant plus remarquable, que notre sociologue ne cite nommément que deux islamologues proprement dits: le Hollandais, Christian Snouck Gronje, et son compatriote et ami, Carl Heinrich Becker, qui fut un moment son étudiant, puis collègue. Friedrich Ulrich ne pouvait donc espérer meilleure aubaine que d'être cité dans l'une des plus importantes «bibles» des sciences sociales.

Chose tout aussi remarquable, ce n'est ni en Allemagne, ni dans les pays anglo-saxons, mais en France que cette thèse, charriée par l'Éthique protestante de Weber, est le plus connue. Ainsi, Georges-Henri Bousquet, par exemple, grand admirateur de Weber et de Sombart, s'y réfère-t-il pour déplorer que «l'Islam n'[ait], hélas (sic!), pas été éclairé par la belle théorie du péché originel.» (Bousquet G.-H., I990, p. 20-2I et n. I7). L’absence, dans l'islam, de ce lapsisme, dont il est question et sur lequel on insiste depuis Kant et, surtout, Hegel, à propos de la formation de la «religion éthique» (Mensching G., I93 I, p. 329-340) est pourtant un lieu commun (Anawati G.-C., I970, 29-40), que Weber avait, du reste, lui aussi souligné (Weber M., 200I, I/22-2, p.435-436).

Bien entendu, c'est surtout lorsque le contexte est islamique et wébérien que réapparaît la distinction prédestination/prédéterminisme du pasteur Ulrich. C'est le cas dans L'Entrepreneur musulman de Gary Tribou, notamment, qui, d'ailleurs, attribue cette distinction à Catherine Colliot-Thélène, qui y aurait abouti «à la lecture des quelques notes de Weber consacrées à l'Islam » (Tribou G., 1999, p. 22). Dans son article sur «La place de l'Islam dans la sociologie des religions de Max Weber», C. Colliot-Thélène aborde, en effet, les 
deux notes introduites par Weber, peu avant sa mort, dans L'Éthique protestante et l'esprit du capitalisme (Colliot-thélène C., 1990, p. 26).

Olivier Carré avait repris, lui aussi, à son compte la distinction wébérienne entre prédestination et prédétermination (Carré O., 1986, p. I47). Cette « découverte» est ajoutée au nombre des critiques dirigées contre Maxime Rodinson, auquel il reproche sa réfutation abusive de Max Weber, notamment en confondant prédestination et prédétermination (Carré O., I986, p. I47). «Découverte», disons-nous, car ces deux notions sont inexistantes dans son précédent article, sur le même sujet pourtant, «À propos de la sociologie weberienne de l’Islam» (Carré O., 1982, p. 199-206), écrit en hommage à Maxime Rodinson. La raison en est tout simplement que le livre de Bryan S. Turner sur Max Weber et l'islam (Turner B. S., 1974), dont l'article est un compte rendu, n’aborde pas du tout le thème de la prédestination dans l'islam.

En revanche, dans La Visionwébérienne del' islam (Schluchter W., 1987), W. Schluchter expose la conception wébérienne de la prédestination islamique et présente, pour la première fois, les grandes lignes de la thèse de Friedrich Ulrich. Schluchter est d'ailleurs le seul à avoir abordé le problème de la prédestination dans l'islam dans cet ouvrage, excepté toutefois les quelques passages de Rudolph Peters dans sa communication sur le fondamentalisme musulman (Peters R., 1987, p. 223-227). Mais, dans la version anglaise, Weber and Islam, le même Peters traitera plus amplement du thème de la prédestination et de l'élection, mais toujours sous son angle de prédilection, le fondamentalisme (Peters R., 1999, p. 205-216).

Il faut noter que Peters n'évoque pas plus dans cette analyse que dans celle de la publication allemande la séparation de Weber et d'Ulrich entre prédestination et prédétermination. On aurait voulu, pourtant, que s'agissant d'un contexte wébérien de prédestination islamique, Peters examine cette distinction, souvent prise pour argent comptant par les lecteurs de Weber. 


\section{CONCLUSION}

Conçue en grande partie pour montrer la suprématie culturelle et sotériologique chrétienne, la thèse du pasteur Friedrich Ulrich ne laisse pas d'étonner par la persistance de certaines de ses formulations dans la littérature consacrée à la vision wébérienne de l'islam, dont celle avant tout donnant le primat à la prédétermination sur la prédestination proprement dite. Reprise sans être discutée, en raison de la caution que lui avait apportée Max Weber lui-même en s'y référant à plusieurs reprises, cette distinction, plus théorique que réelle, ne résiste évidemment pas à la critique. Weber aurait certes tiré le plus grand parti d'une recherche comme celle d'Abraham de Vlieger, par exemple, que le grand ténor de l'islamologie de l'époque, Ignaz Golziher, avait accueilli très favorablement. Naturellement, un tel écart de la part de Max Weber ne signifie nullement la remise en cause de sa démarche. Tout au contraire, sa méthode et ses instruments heuristiques sont à même de mener à une plus grande «compréhension» de l'islam.

\section{BIBLIOGRAPHIE}

ANAWATI Georges Chehata, 1970, «La notion de "péché originel" existe-t-elle dans l'Islam?», Studia Islamica, XXXI, p. 29-40.

BAYDAWI Abdallah, 1846/1848, Commentarius in Coranum, Leipzig, F.C.G. Vogel.

BECKER Carl Heinrich, 1967, Islamstudien. Vom Wesen und Werden der islamischen Welt, Hildersheim, Georg Olms.

BOUSQUET Georges-Henri, 1990, L'éthique sexuelle de I'Islam, Paris,

Desclée de Brouwer.

BUHARI Muhammad ibn Ismaïl, 1908, Sahih, Leyde, E.J. Brill.

CARRÉ Olivier, 1982, «À propos de la sociologie weberienne de l'Islam», in Jean-Pierre Digard (dir.), Le Cuisinier et le Philosophe: hommage à Maxime Rodinson, Paris, Maisonneuve et Larose, p. 199-206.

CARRÉ Olivier, 1986, «À propos de Weber et l'Islam», Archives de Sciences Sociales des Religions, LXI-I, p. I39-152. 
COLLIOT-THÉLÈNE Catherine, 1990, «La place de l'Islam dans la sociologie des religions de Max Weber», Revue du Mauss, n I0, p.6-30.

DJEDI Youcef, 2006, Max Weber et l'Islam, Lyon, ENS.

-, 2007, «Max Weber et le spinozisme ou la "conduite de vie" du "sage"», Le Portique, e-portique 5 - 2007.

-, 2012, «Sociologie et Islamwissenschaft», Der Islam, Bd 86, p. 312-37I.

GOLDZIHER Ignaz, 1910, Vorlesungen über den Islam, Heidelberg, Carl Winter.

-, 1905, «Die Fortschritte der Islam-Wissenschaft in den letzten drei Jahrzehnten », Preußische Jahrbücher, p. 274-300.

-, 1902, «A. de Vliegers Kitâb al-Qadr», Zeitschrift der Deutschen Mortenländischen Gesellschaft, LVI, p. 392-402.

HANISCH Ludmila, 2000, «Machen Sie doch unseren Islam nicht gar zu schlecht». Der Briefwechsel der Islamwissenschaftler Ignaz Goldziher und Martin Hartmann 1894-1914, Wiesbaden, Harrowitz Verlag.

HARNACK Adolf (von), 1964 [1910], Lehrbuch der Dogmengeschichte, Darmstadt, Wissenschaftliche Buchgesellschaft.

HAURI Johannes, I88I, Der Islam in seiner Einfluss auf das Leben seiner Bekenner, Leyde, E.J. Brill.

KREHL Ludolf, 1870, «Über die koranische Lehre von der Prädestination und ihr Verhältnis zu anderen Dogmen des Islâm», Berichte über die Verhandlungen der sächsischen Gesellschaft der Wissenschaften, XXII, n I.

MENSCHING Gustav, 1931, «Typologie außerchristlicher Religionen bei Hegel», Zeitschrift für Missionskunde und Religionswissenschaft, XLVI, p. 329-340.

MIÈGE Jean-Louis, 1955, «Les missions protestantes au Maroc», Hespéris, le-2e trim., p. 153-189.

MUIR William, 1988 [|86|], The Life of Mahomet, Osnabrück, Biblio Verlag.

MÜLLER Gottfried, 1988, «Die Barmherzigkeit Gottes. Zur Entstehungsgeschichte eines koranischen Symbols», Die Welt des Islams, XXVIII, p. 334-362. 
PETERS Rudolph, 1987, «Islamischer Fundamentalismus: Glaube, Handeln, Führung», in Wolfgang Schluchter (dir.), Max Webers Sicht des Islams, Frankfurt a/M, Suhrkamp, p. 223-227.

-, 1999, "Paradise or Hell? The Religious Doctrine of Election in Eighteenth and Nineteenth Century Islamic Fundamentalism and Protestant Calvinism", in Wolfgang Schluchter et Toby E. Huff (dir.), Max Weber and Islam, New Brunswick/London, Transaction Publishers, p. 205-216.

PISCHON C. Nathanael, I88I, Der Einfluss des Islâm auf das häusliche, sociale und politische Leben seiner Bekenner. Eine Culturgeschichtliche Studie, Leipzig, Brockhaus.

RITTER Hellmut, 1977, «L'orthodoxie a-t-elle une part dans la décadence?», in Classicisme et déclin culturel dans I'histoire de l'Islam, Paris, Maisonneuve \& Larose.

RODINSON Maxime, 1966, Islam et Capitalisme, Paris, Maspéro.

SCHLUCHTER Wolfgang (dir.), 1987, Max Webers Sicht des Islams, Frankfurt a/M, Suhrkamp.

-, HUFF Toby E. (dir.), 1999, Max Weber and Islam, New Brunswick/London, Transaction Publishers.

SPIESS Bernhardt, 1873, «Die Prädestinationslehre des Korân», Program des Gymnasiums Weilburg.

TIBAWI Abdallatif, 1963, «The American Missionaries in Beirut and Butrus al-Bustani », St. Anthony's Papers, n 16, p. 137-182.

TRIBOU Gary, 1999, L'Entrepreneur musulman. L'islam et la rationalité d'entreprise, Paris, L'Harmattan.

TURNER Bryan S., 1974, Weber and Islam. A critical Study, London/Henley/Boston, Routledge \& Kegan.

ULRICH Friedrich, 1910, Unsere Neuprotestanten und was wir ihnen schuldig sind, Halle, Verlag des Evangelischen Budes.

-, 1912, Die Vorherbestimmungslehre im Islam und Christentum. Eine religionsgeschichtiliche Parallele, Gütersloh, C. Bertelsmann.

-, 1925, Wohlfartspflege und Persönlichkeit: Wohlfartspflege und Kirche, Berlin Dahlem, Wichern-Verlag. 
-, 1929, Soziale Gerichtshilfe, Berlin - Dahlem, Wichern-Verlag.

-, 1929, Die chistliche Hospize: Festschrift zur Feier des 25jährigen Bestehens des Verbandes christlicher Hospize 1904 - 1929, Berlin, Bertinetti.

-, 1932, Die weltanschaulichen Grundlagen der Wohlfahrtspflege, Berlin, Heymann.

-, 1934, Das Bekenntnis unseres Glaubens, Graz, Graz-Verlag.

-, 1934, Der Gottesdienst für die Kinder, Wien, Evangelische Buchhandlung.

-, 1934, Schöpfer und Schöpfung, Wien, Evangelische Buchhandlung.

-, 1934, Der alte und der neue Weg des evangelischen Religionsunterrichtes, Gratz, Evangelische Buchhandlung.

-, 1935, «Die evangelische Kirche in Österreich», in Friedrich SiegmundSchultze (dir.), Ekklesia: eine Sammlung von Selbstdarstellungen der christlichen Kirchen, Gotha, Klotz.

VLIEGER Abraham (de), 1902, Kitâb al-qadr. Matériaux pour servir à l'étude de la prédestination dans la théologie musulmane, Leyde, E.J. Brill.

WEBER Max, 1988, Gesammelte Aufsätze zur Religionssoziologie, Tübingen, JCB Mohr.

-, 200I-2005, Gesamtausgabe, I, 22/2-4, Tübingen, JCB Mohr.

-, 2003, L'Éthique protestante et l'esprit du capitalisme. Suivi d'autres essais (trad. J.-P. Grossein), Paris, Gallimard. 